Collection SFN 12 (2011) 3-14

(C) Owned by the authors, published by EDP Sciences, 2011

DOI: $10.1051 / \mathrm{sfn} / 201112002$

\title{
Numerical simulations and spectroscopy
}

\author{
F. Guillaume
}

Université Bordeaux 1, UMR CNRS 5255, ISM, Groupe Spectroscopie Moléculaire, 351 cours de la Libération, 33405 Talence Cedex, France

\begin{abstract}
Spectroscopy is the usual tool for analysing dynamic properties of molecular systems at equilibrium. First linear response theory and the fluctuation theorem are introduced here with the example of the Langevin equation describing the Brownian motion. The statistical mechanics concepts issued from this elementary introduction emphasize the links between numerical simulations and spectroscopy. In the second part of this paper we introduce neutron scattering spectroscopy for non-magnetic materials. In particular we discuss on the correlation functions that can be obtained using this technique.
\end{abstract}

\begin{abstract}
La spectroscopie est le moyen d'analyse privilégié pour étudier la dynamique de systems moléculaires à l'équilibre thermodynamique. Nous introduisons ici la théorie de la réponse linéaire et le théorème de fluctuation-dissipation à travers l'exemple simple de l'équation de Langevin décrivant le mouvement Brownien. Les concepts de mécanique statistique qui se dégagent de l'approche de Langevin mettent en valeur les liens profonds qui lient la modélisation numérique et la spectroscopie. Finalement nous présentons brièvement les fonctions de corrélation accessibles en spectroscopie de diffusion neutronique pour des matériaux non magnétiques.
\end{abstract}

\section{INTRODUCTION}

Spectroscopic techniques, including neutron scattering, are the adapted tools to analyse the dynamic properties of a system at a microscopic level. The purpose of this introductory paper is to provide some elementary concepts to bridge the gap between experimental and theoretical approaches. Since the very beginning of spectroscopy, theoretical approaches and modelling and simulations in particular, have always been developed in parallel to technological improvements for interpreting experimental data obtained on chemical systems of increasing complexity.

From the theoretical side, we will discuss first on the Linear Response Theory (LRT) on the basis of the analysis of the Brownian motion. LRT contains all the necessary concepts to understand how spectroscopic means allow the measurement of dynamic properties of molecules for a real system at equilibrium. Indeed the fluctuation dissipation theorem links the output of an experiment to the spontaneous fluctuations of molecules or particles for a system at equilibrium.

From the point of view of the experimentalist, the molecular properties of a system are analyzed through its spectrum. As emphasized above, spectroscopy and computational techniques are highly complementary. This is particularly true for neutron scattering and we will show that from a model of atomic positions as a function of time, it is easy to calculate the expected neutron scattering spectrum and to compare it to experimental results.

This is an Open Access article distributed under the terms of the Creative Commons Attribution-Noncommercial License 3.0, which permits unrestricted use, distribution, and reproduction in any noncommercial medium, provided the original work is properly cited. 


\section{SOME CONCEPTS IN LINEAR RESPONSE THEORY}

As a guide for the discussion on LRT, we will follow the famous description of the Brownian motion proposed by Paul Langevin. Robert Brown in 1828 first described the perpetual and irregular motion of pollen grains in a liquid. The importance of this discovery has soon been recognized but was a mystery in physics, many experiments have proven that no external influences could be at the origin of the erratic nature of the Brownian motion. It is much later that precursor scientists, among others J. Delsaux in 1877 and G.L. Gouy in 1888, formulated the hypothesis that the continuous motion of particles suspended in a fluid was due to the molecular thermal agitation [1]. About 20 years later, in 1905, Albert Einstein [2] showed that Brownian motion could be understood in terms of choc of molecules. The Einstein's equations, based on the molecular-kinetic theory of heat, were verified experimentally by Jean Perrin [3] in 1908 proving the reality of molecules. At the same time Paul Langevin published a short note [4] on the theory of Brownian motion. The key concepts of Langevin's approach, quite revolutionary at this time although apparently very simple, contributed to inspire the new field of stochastic processes.

Many excellent books or reviews dealing with LRT have been published with some of them being particularly accessible for students [5-8].

\subsection{The Brownian motion}

Paul Langevin simply wrote the equation of motion for a particle in a fluid at equilibrium according to Newton's laws. However, he introduced for the first time the concept of a random force acting on the Brownian particle due to the collisions of the surrounding molecules and a viscous force proportional to the particle's velocity (Stokes' law). To illustrate some important concepts of LRT, we add a weak external force to the Langevin equation for modelling a system weakly perturbed, still close to equilibrium.

\subsubsection{The Langevin equation for the system at equilibrium}

For the system at equilibrium, we assume that the total force acting at time $\mathrm{t}$ on the Brownian particle is the sum of a frictional force $-\gamma \vec{v}(t)$ and a fluctuating force $\vec{F}(t)$. The equation of motion for the Brownian particle, i.e. the Langevin equation, is therefore:

$$
m \frac{d \vec{v}(t)}{d t}=-\gamma \vec{v}(t)+\vec{F}(t)
$$

For the system at equilibrium the mean velocity at any time (ensemble average) is zero. Assumptions on the fluctuating force are necessary to solve the (stochastic) differential equation (1). First of all the average of $\vec{F}(t)$ is zero so that at any time $t$ (including initial time $t=0$ ), we have:

$$
\langle\vec{v}(t)\rangle=\overrightarrow{0} \text { and }\langle\vec{F}(t)\rangle=\overrightarrow{0}
$$

Second, the force $\vec{F}(t)$ varies very quickly compared to the variations of $\vec{v}(t)$, the collisions are instantaneous, so that the fluctuating force is not correlated to the velocity of the Brownian particle and the correlation function $\left\langle\vec{F}(t) \vec{F}\left(t^{\prime}\right)\right\rangle$ is nonzero only if $t^{\prime}=t$ :

$$
\left\langle\vec{F}(t) \vec{F}\left(t^{\prime}\right)\right\rangle=\Gamma \cdot \delta\left(t-t^{\prime}\right) \text { and }\left\langle\vec{v}(t) \vec{F}\left(t^{\prime}\right)\right\rangle=\langle\vec{v}(t)\rangle\left\langle\vec{F}\left(t^{\prime}\right)\right\rangle=0
$$

The solution of equation (1) is:

$$
\vec{v}(t)=\vec{v}(0) \cdot e^{-\frac{\gamma}{m} t}+\frac{1}{m} \int_{0}^{t} \vec{F}\left(t^{\prime}\right) \cdot e^{-\frac{\gamma}{m}\left(t-t^{\prime}\right)} d t^{\prime}
$$

Now taking the average of (4) we get $\langle\vec{v}(t)\rangle=\overrightarrow{0}$, as expected. The autocorrelation function for the velocity can be obtained first by multiplying $\vec{v}(t)$ by $\vec{v}(t+\tau)$ and second by averaging. The stationary 
solution is:

$$
\left\langle\vec{v}(t) \vec{v}(t+\tau)=\frac{\Gamma}{2 m \gamma} e^{-\frac{\gamma}{m} \tau}\right.
$$

According to the equipartition theorem $\left(\left\langle v^{2}\right\rangle=\frac{3 k T}{m}\right)$, we get $\Gamma=6 \gamma k T$. We can see that the velocity autocorrelation function for the system at equilibrium depends only on $\tau$, with $\frac{m}{\gamma}$ the correlation time for the Brownian motion. It is interesting here to note that $\Gamma$ is proportional to the friction term $\gamma$ so that:

$$
\gamma=\frac{1}{6 k T} \int_{0}^{\infty}\langle\vec{F}(t) \vec{F}(t+\tau)\rangle d \tau
$$

This equation (6) relates the systematic frictional force to the random force and is a form of the so-called "second fluctuation-dissipation theorem".

\subsubsection{The response function}

Let us now consider the Langevin equation by adding an external force $\vec{F}_{\text {ext }}(t)$ (the perturbation) to the system of Brownian particles. Our goal is now to analyze their behaviour (the response) for the system put slightly out of equilibrium.

At some initial time $\mathrm{t}=0$ the system is at equilibrium. We apply an instantaneous external force on all the Brownian particles at time $t_{0}>0$. Of course equations (2) and (3) hold for $0 \leq t<t_{0}$ and $\vec{F}_{\text {ext }}(t)$ writes as follows:

$$
\vec{F}_{\text {ext }}(t)=\vec{F}_{0} \cdot \delta\left(t-t_{0}\right)
$$

Solving the Langevin equation (1) with this additional term gives:

$$
\begin{aligned}
\langle\vec{v}(t)\rangle & =\frac{\vec{F}_{0}}{m} e^{-\frac{\gamma}{m}\left(t-t_{0}\right)} \quad \text { for } t \geq t_{0} \\
& =\overrightarrow{0} \text { for } t<t_{0}
\end{aligned}
$$

The time taken for the system to re-establish equilibrium, the relaxation time, is the integral of equation (8), i.e. simply the correlation time $\frac{m}{\gamma}$ defined for the system at equilibrium. We may use another way of writing equation (8):

$$
\langle\vec{v}(t)\rangle=\int_{-\infty}^{\infty} \vec{F}_{\text {ext }}\left(t^{\prime}\right) \cdot \Phi\left(t-t^{\prime}\right) d t^{\prime} \equiv \vec{F}_{\text {ext }}(t) \otimes \Phi(t)
$$

Where

$$
\Phi(t)=\frac{1}{m} e^{-\frac{\gamma}{m} t}
$$

is the response function of the system. Note that to obtain equation (9) it is necessary to assume the causality principle allowing substituting the time $\mathrm{t}$ by infinity and the origin by minus infinity in the integration limits. Equation (9) is in fact very general (see more rigorous treatments of the linear response theory in another paper of this book [9]), i.e. a measurable property of the system (here the mean velocity) is the convolution product of the excitation and the response function. For the Brownian motion, we may refer to equation (6) to write:

$$
\Phi(\tau)=\frac{1}{3 k T}\langle\vec{v}(t) \vec{v}(t+\tau)\rangle
$$

In other words the property measured by setting the system out of equilibrium is directly related to its autocorrelation function at equilibrium, i.e. without any external perturbation! 


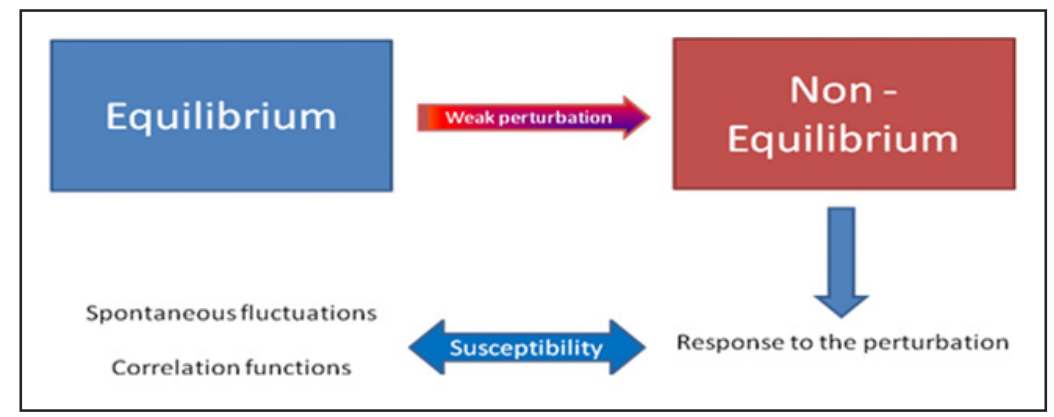

Figure 1. Linear Response Theory: how the system put out of equilibrium by an external perturbation can provide information on the spontaneous fluctuations of the molecules in the absence of any perturbation.

\subsection{The harmonic response: susceptibility}

We now consider a harmonic external force so that:

$$
\vec{F}_{\text {ext }}(t)=\vec{F}_{0} \cdot \Re e^{i 2 \pi v t}
$$

It comes out that, by applying relations (9) and (10), the mean velocity of the Brownian particle out of equilibrium writes as:

$$
\langle\vec{v}(t)\rangle=\Re\left(\vec{F}_{0} \cdot e^{i 2 \pi v t} \cdot \int_{-\infty}^{\infty} \frac{1}{m} e^{-\gamma t^{\prime}} \cdot e^{i 2 \pi v t^{\prime}} d t^{\prime}\right)
$$

Equation (13) is the product of the excitation by the Fourier transform of the response function called, in the frequency domain, the susceptibility. The susceptibility is complex and can be written as:

$$
\chi(v)=\frac{1}{3 m k T} \int_{-\infty}^{\infty}\langle\vec{v}(0) \vec{v}(\tau)\rangle \cdot e^{-i 2 \pi v \tau} d \tau \equiv \frac{1}{m} \cdot \frac{1}{\gamma+i 2 \pi v}
$$

Thus equation (13) can be written, using (14):

$$
\langle\vec{v}(t)\rangle=\Re\left(\chi(v) \cdot \vec{F}_{\text {ext }}(t)\right)
$$

Equation (15) shows that the property induced by applying an external force to the system is proportional to this force (it is by definition a linear response), the coefficient of proportionality is the susceptibility. For example applying an oscillating electric field in a solution containing electric dipoles will produce a polarisation proportional to the applied field. The susceptibility is complex, i.e. $\chi(v)=\chi^{\prime}(v)-i \chi^{\prime \prime}(v)$ and it can be shown [5-8] that the dissipated power is proportional to $\chi^{\prime \prime}(v)$, i.e. there is a direct connection between dissipation and susceptibilities. Moreover the susceptibility involves fluctuations in equilibrium that were present without applying the external field. This result is actually quite general and we will identify the imaginary part of the susceptibility with the absorption of energy. The "fluctuationdissipation theorem" (Fig. 1) is a result which relates the linear response coefficients (which describe, among other things, dissipative effects and the irreversible approach of a system to equilibrium) to simpler correlation functions describing fluctuations which occur in the system in equilibrium.

\subsection{Spectroscopy}

All the concepts described qualitatively in the preceding paragraph apply of course to merely all spectroscopic techniques. Properties of systems at equilibrium can be obtained by applying a weak perturbation inducing a measurable property. As already mentioned above, applying an oscillating electric field to a dielectric system will induce the displacement of charges and therefore a macroscopic 
polarisation. Two major phenomena will be observed [10], one is the absorption and the other is the scattering of light. In both cases the experimentally obtained quantity is the imaginary part of the susceptibility and therefore the Fourier Transform of a correlation function.

Let us for example consider an isotropic liquid of identical polar molecules diluted in an apolar solvent. Now we put this sample in a beam with wavelengths in the microwave region (wavelengths in the range $1 \mathrm{~mm}$ to $1 \mathrm{~cm}$ ). In the microwave range the incoming beam is partially absorbed because all the molecular dipoles couple to the electric field. Thus the electric polarisation of the medium is proportional to the external field and the corresponding electric susceptibility (or permittivity) will be the Fourier Transform of the correlation functions $\Psi(t)$ of the electric moments $\vec{M}(t)$ :

$$
\Psi(t)=\langle\vec{M}(0) \vec{M}(t)\rangle \quad \text { with } \quad \vec{M}(t)=\sum_{i=1}^{N} \vec{\mu}_{0}^{i}(t)
$$

Where $\vec{\mu}_{0}^{i}(t)$ is the permanent dipole moment of molecule $\mathrm{i}$ at time t. Thus we may write:

$$
\Psi(t)=\left\langle\sum_{i=1}^{N} \vec{\mu}_{0}^{i}(t) \sum_{j=1}^{N} \vec{\mu}_{0}^{j}(t)\right\rangle \equiv N\left\langle\vec{\mu}_{0}(0) \vec{\mu}_{0}(t)\right\rangle+\sum_{\substack{i, j=1 \\ i \neq j}}^{N}\left\langle\vec{\mu}_{0}^{i}(0) \vec{\mu}_{0}^{j}(t)\right\rangle
$$

In this equation $\left\langle\vec{\mu}_{0}(0) \vec{\mu}_{0}(t)\right\rangle$ is the "self" correlation function of the dipole moments and $\sum_{\substack{i, j=1 \\ i \neq j}}^{N}\left\langle\vec{\mu}_{0}^{i}(0) \vec{\mu}_{0}^{j}(t)\right\rangle$ is the "cross" correlation function. Obviously the "self" correlation function describes the mean fluctuations of one dipole at equilibrium and the "cross" correlation function describes collective dynamic properties. As the dipole moment is collinear to the highest symmetry axis of the polar molecule, dielectric spectroscopy will provide information on the orientation fluctuations of the molecules for the system at equilibrium. We may also note that for a dilute solution of dipolar molecules in an apolar solvent, the "cross" correlation function in equation (17) vanishes as the orientations of different molecules are not correlated to each other.

We summarize in table 1 the correlation functions measured using some optical spectroscopic techniques.

\section{NEUTRON SPECTROSCOPY}

In this section we introduce the neutron scattering technique. Our goal is to underline some general principles of this spectroscopy. For further reading we recommend strongly the excellent review of H. Schober in a previous volume of the series "SFN collection" [11] and also the book of M. Bée [12] about quasielastic neutron scattering.

\subsection{The neutron scattering law}

We consider now the scattering of neutrons due to the nuclear interaction with the nuclei of the sample as schematized in Fig. 2. In this introduction we will not consider magnetic systems. The neutron possesses properties highly interesting for investigating dynamics in condensed matter. First of all the neutron energy may be tuned to match the translational, rotational or vibrational energy levels. In addition the wavelength of the corresponding neutron field is short, of the order of atomic or inter-molecular distances. Thus, in contrast to optical spectroscopy, neutron spectroscopy will probe fluctuations both in time and space domains. The interaction at time t between a neutron located at $\vec{r}$ and a nucleus i with scattering length $b_{i}$ at position $\vec{R}_{i}(t)$ is described by means of the Fermi pseudo potential $V_{i}(\vec{r}, t)$ that represents the very short range neutron-nucleus nuclear interaction:

$$
V_{i}(\vec{r}, t)=\frac{2 \pi \hbar^{2}}{m} b_{i} \delta\left(\vec{r}-\vec{R}_{i}(t)\right)
$$


Table 1. Correlation functions probed by means of some optical spectroscopic techniques.

\begin{tabular}{|c|c|c|}
\hline Technique & Coupling variable & Correlation function \\
\hline $\begin{array}{l}\text { Dielectric relaxation } \\
\text { (absorption) }\end{array}$ & Permanent dipole moment $\vec{\mu}_{0}$ & $\begin{array}{c}N\left\langle\vec{\mu}_{0}(0) \cdot \vec{\mu}_{0}(t)\right\rangle \\
+\sum_{\substack{i, j=1 \\
i \neq j}}^{N}\left\langle\vec{\mu}_{0}^{i}(0) \cdot \vec{\mu}_{0}^{j}(t)\right\rangle\end{array}$ \\
\hline Infrared absorption $^{(1)}$ & $\begin{array}{l}\text { Induced dipole moment }{ }^{(3)} \\
\qquad\left(\frac{\partial \vec{\mu}(t)}{\partial q}\right)_{0} \cdot q(t)\end{array}$ & $\left\langle\left(\frac{\partial \vec{\mu}(0)}{\partial q}\right)_{0} \cdot\left(\frac{\partial \vec{\mu}(t)}{\partial q}\right)_{0}\right\rangle \cdot\langle q(0) \cdot q(t)\rangle$ \\
\hline Rayleigh scattering & Polarisablility tensor $\overleftrightarrow{\alpha}_{0}(t)$ & $\begin{array}{c}N\left\langle\overleftrightarrow{\alpha}_{0}(0) \cdot \overleftrightarrow{\alpha}_{0}(t)\right\rangle \\
+\sum_{\substack{i, j=1 \\
i \neq j}}^{N}\left\langle\overleftrightarrow{\alpha}_{0}^{i}(0) \overleftrightarrow{\alpha}_{0}^{j}(t)\right\rangle\end{array}$ \\
\hline $\operatorname{Raman}$ Scattering ${ }^{(1,2)}$ & $\begin{array}{l}\text { Induced polarisability tensor } \\
\qquad\left(\frac{\partial \overleftrightarrow{\alpha}(t)}{\partial q}\right)_{0} \cdot q(t)\end{array}$ & $\left\langle\left(\frac{\partial \overleftrightarrow{\alpha}(0)}{\partial q}\right)_{0} \cdot\left(\frac{\partial \overleftrightarrow{\alpha}(t)}{\partial q}\right)_{0}\right\rangle \cdot\langle q(0) \cdot q(t)\rangle$ \\
\hline
\end{tabular}

(1) Assuming no correlations between the fluctuations of the vibrational normal coordinate $q(\mathrm{t})$ and the orientation of the molecule at time $\mathrm{t}$ and also no correlations between the vibrations of molecule $i$ and the vibrations of molecule $j$.

(2) Note that in the case of Raman scattering by an isotropic system, the polarisation states of the incoming laser beam and of the scattered radiation may be controlled. Thus an isotropic spectrum can be obtained that depends only on $\langle q(0) \cdot q(t)\rangle$ and an anisotropic one depending upon $\left\langle\left(\frac{\partial \overleftrightarrow{\beta}(0)}{\partial q}\right)_{0} \cdot\left(\frac{\partial \overleftrightarrow{\beta}(t)}{\partial q}\right)_{0}\right\rangle \cdot\langle q(0)$.

$q(t)\rangle$ where $\stackrel{\leftrightarrow}{\beta}$ is the anisotropy of the polarisability tensor $\overleftrightarrow{\alpha}$.

(3) $\mathrm{q}(t)$ represents the normal coordinate of vibration at time $t$.

where $m$ is the mass of the neutron. The scattering of the neutron is isotropic and the scattering length $b_{i}$ is a complex number, its real part is negative for a repulsive interaction and positive for an attractive interaction whereas the imaginary part is the probability of absorption of the neutron by the nucleus. Furthermore, the scattering length depends on the relative spin orientation of the nucleus $v s$ that of the neutron, i.e. on the total spin state of the nucleus-neutron system. The scattering lengths are experimentally known (tabulated in reference [13]) and for a system with many nuclei the Fermi pseudo potential will be given by the sum of the individual contributions of equation (18). In a neutron scattering experiment schematized in Fig. 2, a monochromatic beam of neutrons (with energy $\hbar \omega_{0}$ and wave vector $\vec{k}_{0}$ ) impacts the sample. Scattered neutrons with wave vector $\vec{k}^{\prime}$ and energy $\hbar \omega^{\prime}$ are analysed as a function of their energy transfer $\hbar \omega=\hbar \omega^{\prime}-\hbar \omega_{0}$ and momentum transfer $\hbar \vec{Q}=\hbar\left(\vec{k}^{\prime}-\vec{k}_{0}\right)$.

The experimentally available quantity, for a system containing many different atoms, is thus the double differential cross-section that represents the number of neutrons scattered in the solid angle element $d \Omega$ with energy between $E$ and $E+d E$ :

$$
\frac{d^{2} \sigma}{d \Omega d E}=\frac{k}{k_{0}} S(\vec{Q}, \omega)
$$

where $S(\vec{Q}, \omega)$ is called the scattering law. Of course $S(\vec{Q}, \omega)$ can be identified to the imaginary part of the susceptibility of the system. Therefore the relevant correlation function to consider in neutron scattering is, by a crude analogy with the Brownian motion discussed in section 2.2, the inverse Fourier Transform in the time $(t)$ and space $(\vec{r})$ domains of the scattering law. The coupling variable $\delta\left(\vec{r}-\vec{R}_{i}(t)\right)$ between the neutron and the system given in equation (18) is the dynamic variable experimentally available in neutron spectroscopy. The correlation function to be considered is the pair correlation function $G_{i, j}(\vec{r}, t)$ that writes as [12]:

$$
G_{i, j}(\vec{r}, t)=\int\left\langle b_{i} b_{j} \delta\left(\vec{r}-\vec{r}^{\prime}+\vec{R}_{i}(0)\right) \delta\left(\vec{r}^{\prime}-\vec{R}_{j}(t)\right)\right\rangle d \vec{r}^{\prime}
$$




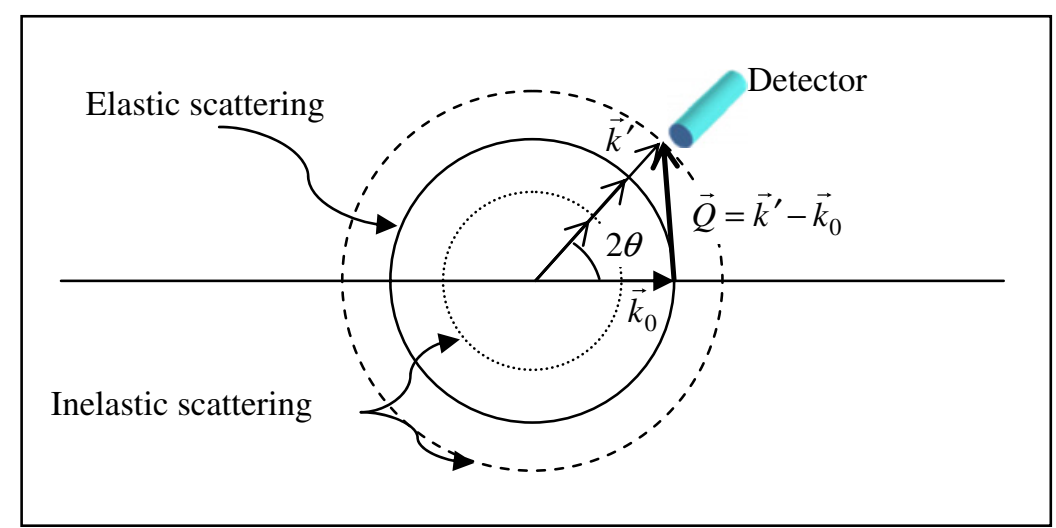

Figure 2. The sample is irradiated by a monochromatic neutron beam with incident wave vector $\vec{k}_{0}$. The scattering of the neutron may be elastic $\left(k^{\prime}=k_{0}\right)$ or inelastic with energy gain $\left(k^{\prime}>k_{0}\right)$ or energy loss $\left(k^{\prime}<k_{0}\right)$.

Note the position vectors are quantum mechanical position operators. However, for simplicity, we can treat the vectors as classical quantities. The Fourier Transform of $G_{i, j}(\vec{r}, t)$ in the space domain gives the intermediate scattering function $I_{i, j}(\vec{Q}, t)$ :

$$
I_{i, j}(\vec{Q}, t)=\left\langle b_{i} b_{j} e^{i \vec{Q} \vec{R}_{1}(0)} \cdot e^{-i \vec{Q} \vec{R}_{j}(t)}\right\rangle
$$

And finally the Fourier Transform of $I_{i, j}(\vec{Q}, t)$ in the time domain gives the scattering law in equation (19):

$$
S(\vec{Q}, \omega)=\frac{1}{2 \pi N} \sum_{i, l} \int_{-\infty}^{+\infty} I_{i, j}(\vec{Q}, t) \cdot e^{-i \omega t} d t
$$

\subsection{Incoherence and coherence}

The specimen is composed of $\mathrm{N}$ atomic species, each of them being composed of several isotopes in different spin states. Because there is no correlation between the spin of a nucleus and its position, the scattering lengths appearing in equation (21) can be averaged without interfering with the thermal average denoted by the angular brackets. The mean values $\left\langle b_{i}\right\rangle$ and $\left\langle b_{i}^{2}\right\rangle$ can be calculated over all isotopes and spin states. The incoherent and coherent scattering lengths $b^{i n c}$ and $b^{c o h}$ are defined by:

$$
b^{i n c}=\sqrt{\left[\left\langle b_{i}^{2}\right\rangle-\left\langle b_{i}\right\rangle^{2}\right]} \text { and } b^{\text {coh }}=\left\langle b_{i}\right\rangle
$$

and, as demonstrated in detail in references [11] and [12], we get:

$$
\left\langle b_{i} b_{j}\right\rangle=\left(b^{c o h}\right)^{2}+\left(b^{i n c}\right)^{2} \delta_{i j}
$$

where $\delta_{i j}$ is the Kronecker symbol. The corresponding bound cross sections are defined as $\sigma=4 \pi b^{2}$. For example we can consider a single isotope with nuclear spin s. There are two possible spin states $\mathrm{s}$ $+\frac{1}{2}$ and $\mathrm{s}-\frac{1}{2}$ and we get:

$$
\left\langle b_{i}\right\rangle=\frac{1}{2 s+1}\left[(s+1) b^{+}+s b^{-}\right] \quad \text { and } \quad\left\langle b_{i}^{2}\right\rangle=\frac{1}{2 s+1}\left[(s+1)\left(b^{+}\right)^{2}+s\left(b^{-}\right)^{2}\right]
$$

Where $b^{+}$and $b^{-}$are the scattering lengths associated to the two possible spin states. We have reported in table 2 the scattering lengths for some atoms and comments will follow when considering these values. 
Table 2. Scattering lengths [13] and scattering cross sections for some atoms.

\begin{tabular}{|l|c|c|c|c|}
\hline Element & $b^{+} / \mathrm{fm}$ & $b^{-} / \mathrm{fm}$ & $\sigma^{\text {coh }} /$ barns & $\sigma^{\text {inc }} /$ barns \\
\hline${ }^{1} \mathrm{H}(\mathrm{s}=1 / 2)$ & 1.08 & -4.75 & 1.76 & 80.27 \\
\hline${ }^{2} \mathrm{H}(\mathrm{s}=1)$ & 0.95 & 0.97 & 5.59 & 2.05 \\
\hline${ }^{12} \mathrm{C}(\mathrm{s}=0)$ & 6.65 & & 5.56 & 0 \\
\hline${ }^{16} \mathrm{O}(\mathrm{s}=0)$ & 5.803 & & 4.23 & 0 \\
\hline${ }^{14} \mathrm{~N}(\mathrm{~s}=1)$ & 10.78 & 6.54 & 11.03 & 0.50 \\
\hline${ }^{51} \mathrm{~V}(\mathrm{~s}=5 / 2)$ & 4.96 & -7.91 & 0.02 & 5.07 \\
\hline
\end{tabular}

Thus for a single element the incoherent neutron scattering may therefore be defined as:

$$
S^{i n c}(\vec{Q}, \omega)=\frac{1}{2 \pi N}\left(b^{i n c}\right)^{2} \sum_{i} \int_{-\infty}^{+\infty}\left\langle e^{i \vec{Q} \vec{R}_{i}(0)} \cdot e^{-i \vec{Q} \vec{R}_{i}(t)}\right\rangle \cdot e^{-i \omega t} d t
$$

And the coherent neutron scattering writes:

$$
S^{c o h}(\vec{Q}, \omega)=\frac{1}{2 \pi N}\left(b^{c o h}\right)^{2} \sum_{i, j}^{i \neq j} \int_{-\infty}^{+\infty}\left\langle e^{i \vec{Q} \vec{R}_{i}(0)} \cdot e^{-i \vec{Q} \vec{R}_{i}(t)}\right\rangle \cdot e^{-i \omega t} d t
$$

When considering different nuclei in a sample, the coherent and incoherent scattering lengths are calculated according to equations (23) and (25) taking into account the relative abundances of the atomic and isotopic species $[12,14]$.

Returning now to table 2, one can see that compared to all other nuclei, hydrogen is a strong incoherent scatterer and a weak coherent scatterer. For instance in an organic material with H, N, C or $\mathrm{O}$ atoms, the overall scattering will be essentially incoherent. Moreover this scattering will be due to the individual displacements of hydrogen only. For carbon or oxygen atoms with $\mathrm{s}=0$, the scattering will be exclusively coherent, i.e. only collective properties will be available from experiments. For organic compounds neutron scattering will be mostly incoherent. For example (see table 2 ) the total cross section for benzene is 89.67 barns and the incoherent cross section is 82.32 barns. The incoherent scattering for the hydrogen atoms of benzene represents $89.52 \%$ of the total scattering!

Finally, let us emphasize that there are no proper "selection rules" in neutron spectroscopy, in contrast to other techniques such as infrared absorption or Raman scattering with selection rules determined by the symmetry properties of the system. All vibration modes will be active in neutron scattering. Also long range translations do not involve changes in the molecular polarisability tensor or dipole moment whereas neutron scattering probes the displacements of atoms. Clearly neutron spectroscopy can provide information on rotational, translational and vibration fluctuations in matter and a difficulty might be to disentangle all these processes. In this case computational methods will help significantly.

\subsection{Elastic and inelastic scattering}

\subsubsection{Elastic scattering}

In elastic neutron scattering the kinetic energy of the incident particles is conserved and only their direction of propagation is modified. Thus the scattering law in equation (22) is a function of the scattering vector $\vec{Q}$ only. To illustrate elastic scattering we consider a very simple example of a $1-\mathrm{D}$ crystal with one atom per unit cell and primitive vector $\vec{a}$ (see Fig. 3). For elastic scattering at $\hbar \omega=0$, we get from equations (22), (26) and (27):

$$
S(\vec{Q})=\frac{N b^{c o h^{2}}}{2 \pi} \cdot \sum_{a^{*}} \delta\left(\vec{Q}-\vec{a}^{*}\right)+\frac{b^{i n c^{2}}}{2 \pi \hbar}
$$




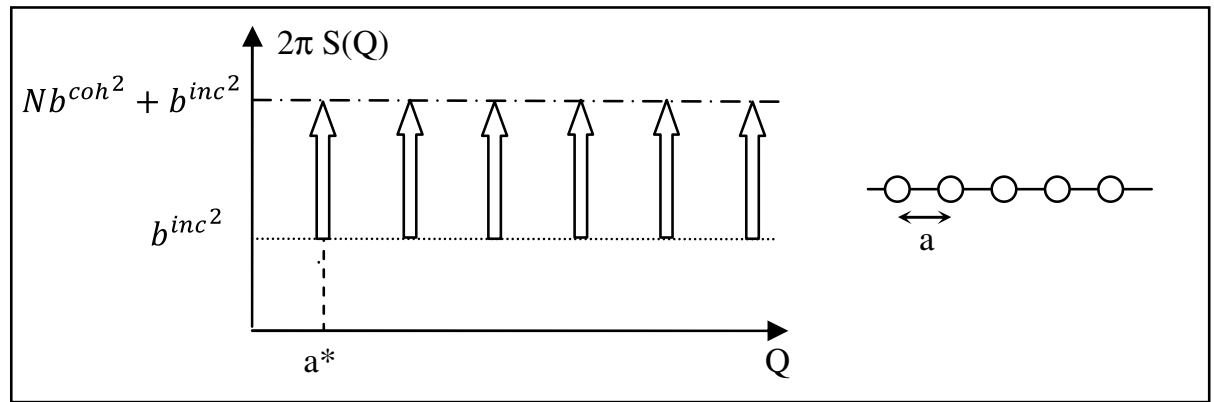

Figure 3. Schematic representation of the elastic neutron scattering in equation (28): the coherent scattering (Bragg peaks) is symbolized by the arrows and the incoherent scattering by the dotted line.

Equation (28) is shown in Fig. 3. The coherent elastic scattering function represents the diffraction peaks along the reciprocal axis $a^{*}$ providing information on the structure of the crystal whereas the incoherent scattering is a constant "background".

\subsubsection{Vibrations (inelastic scattering)}

We consider now the inelastic scattering for one atom oscillating about a fixed position. We introduce the small amplitude harmonic displacements $\overrightarrow{u_{i}(t)}=\vec{u}_{0} \cos \left(\omega_{0} t\right)$ in equations (21-22) and the series expansion of the intermediate scattering function [11] gives:

$$
I(\vec{Q}, t)=e^{-Q^{2}\left\langle u_{0}^{2}\right\rangle}\left(1+Q^{2}\left\langle u_{0}^{2}\right\rangle \cos \left(\omega_{0} t\right)\right)
$$

So that the scattering for such atom, incoherent by definition, is:

$$
S^{i n c}(\vec{Q}, \omega)=e^{-Q^{2}\left\langle u_{0}^{2}\right\rangle}\left[\delta(\omega)+\frac{Q^{2}\left\langle u_{0}^{2}\right\rangle}{2}\left(\delta\left(\omega-\omega_{0}\right)+\delta\left(\omega+\omega_{0}\right)\right)\right]
$$

This result may be easily generalized to a molecule with $\mathrm{N}$ atoms (sum over $3 \mathrm{~N}-6$ vibration modes). For the chain of atoms described above but coupled to each other by a spring (chain of coupled harmonic oscillators), it is well known that three acoustic branches can be determined with the dispersion relation:

$$
\omega_{0}(q)^{2}=\frac{4 C}{m} \cdot \sin ^{2}\left(\frac{q a}{2}\right)
$$

where $\mathrm{C}$ is the force constant, $\mathrm{m}$ is the mass of the atom and $\vec{q}$ is the phonon wave-vector. The inelastic coherent scattering law will take the following form:

$S^{c o h}(\vec{Q}, \omega)=e^{-Q^{2}\left(u_{0}^{2}\right)}\left[\frac{(\vec{Q}, \vec{u})^{2}}{2}\left(\delta\left(\omega-\omega_{0}(q)\right) \cdot \delta(\vec{Q}-\kappa-\vec{q})+\delta\left(\omega+\omega_{0}(q)\right) \cdot \delta(\vec{Q}-\vec{\kappa}+\vec{q})\right)\right]$

where $\vec{q}$ is the phonon wave-vector and $\vec{\kappa}$ the lattice wave-vector. These results (equations (30) and (32)) are summarized in Fig. 4.

\subsubsection{Translations and rotations (quasielastic scattering)}

We consider now the displacements of one atom due to the rotational and translational motions of a molecule. The motions are not oscillatory so that the inelastic scattering due to translations and rotations will be centred on the zero-energy transfer spectral window; the scattering is named "quasielastic". The incoherent intermediate scattering function $\operatorname{Irt}_{r t}^{\text {inc }}(\vec{Q}, t)$ for translations and rotations can be separated in 


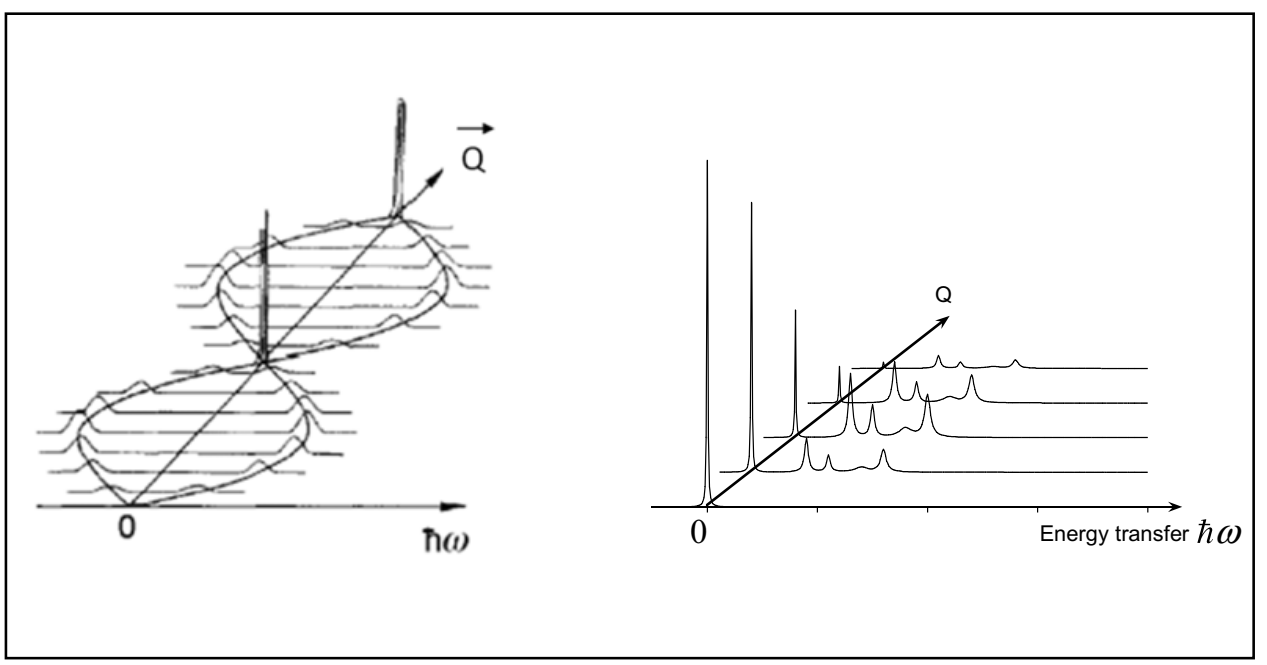

Figure 4. Schematic representation of the coherent (left) and incoherent (right) inelastic neutron scattering in equations (30) and (32).

two terms, a "static" one $I_{r t}^{\text {inc }}(\vec{Q}, \infty)$ called Elastic Incoherent Structure Factor (EISF) and a "timedependent" one $I_{r t}^{t d}(\vec{Q}, t)$ :

$$
I_{r t}^{i n c}(\vec{Q}, t)=I_{r t}^{i n c}(\vec{Q}, \infty)+I_{r t}^{t d}(\vec{Q}, t)
$$

The EISF may also be written as:

$$
I_{r t}^{i n c}(\vec{Q}, \infty)=\left|\left\langle e^{-i \vec{Q} \vec{r}}\right\rangle\right|^{2} \equiv\left|\int d^{3} \vec{r} \cdot G(\vec{r}) e^{-i \vec{Q} \vec{r}}\right|^{2}
$$

where $G(\vec{r})$ is the distribution function of the atomic positions. Thus the EISF represents the space Fourier Transform of the volume accessed by the atom, i.e. gives us information about the configurational space accessible by the scattering atoms. It is interesting to note that the EISF in (34) can be calculated directly from the distribution functions obtained by numerical simulations and compared to the experiments. In a liquid this volume tends to infinity so that the EISF vanishes for all values of $\vec{Q}$. Thus there is no $\delta(\omega)$ elastic peak in the Fourier transform of equation (33). For example the scattering law for a model of translational diffusion (Fick's law) writes as:

$$
S_{\text {Trans }}^{\text {inc }}(Q, \omega)=\frac{1}{\pi} \frac{D Q^{2}}{\omega^{2}+\left(D Q^{2}\right)^{2}}
$$

The width of the quasielastic profile is directly proportional to the diffusion coefficient $\mathrm{D}$ (see Fig. 5a left).

For a solid the EISF is a function of $\vec{Q}$ and the shape of this function will provide information on the geometry of the motions. For example the scattering law for a model of uniaxial rotations by jumps on $\mathrm{N}$ sites equally spaced on a circle of radius $r_{g}$ writes, after powder averaging, as:

$$
\begin{aligned}
& S_{R o t}^{\text {inc }}(Q, \omega)=A_{0}(Q) \cdot \delta(\omega)+\sum_{j=1}^{N-1} A_{j}(Q) \cdot \frac{1}{\pi} \frac{\tau_{j}^{-1}}{\omega^{2}+\tau_{j}^{-2}} \\
& \text { with } A_{j}(Q)=\frac{1}{N} \sum_{i=1}^{N} j_{0}\left(2 Q r_{g} \sin \frac{i \pi}{N}\right) \cos \left(\frac{2 j i \pi}{N}\right) \quad \text { and } \tau_{j}^{-1}=2 \tau^{-1} \sin ^{2}\left(\frac{\pi j}{N}\right) .
\end{aligned}
$$




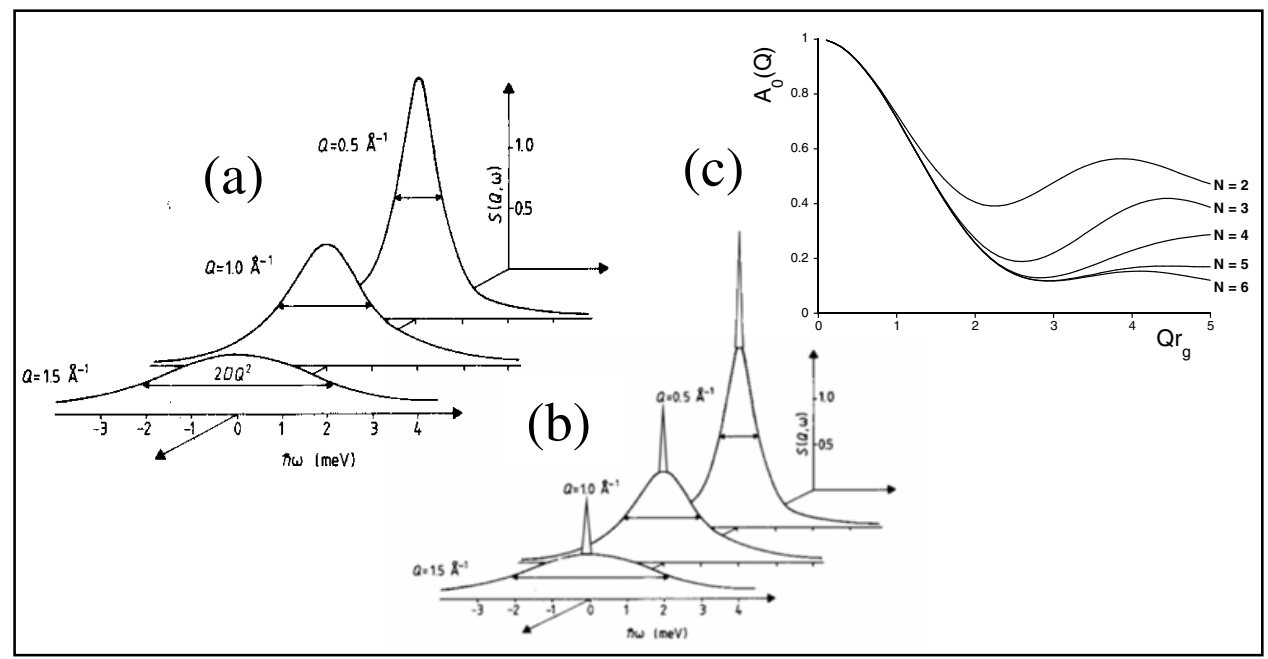

Figure 5. Schematic representation of: the incoherent quasielastic scattering (a) for a liquid, (b) for a solid (right) and (c) the EISFs $A_{0}(Q)$ for the jump model in equation (36).

We have reported in Fig. 5 (right) the typical shape of the quasielastic profile and the differences in the EISFs $A_{0}(Q)$ in equation (35) for values of $\mathrm{N}$ (number of sites) ranging from 2 to 6 . The EISFs are all similar for $Q r_{g}<1 \AA^{-1}$ and can be easily distinguished for larger values of $Q r_{g}$.

We should note that in an incoherent quasielastic neutron scattering experiment, vibrations, rotations and translations do contribute to the total scattering. Combining these motions and assuming that there are no correlations between molecular vibrations and diffusive motions, we get:

$$
I_{q e}(\vec{Q}, t)=I_{v i b}(\vec{Q}, t) \cdot I_{r t}^{i n c}(\vec{Q}, t)
$$

where $I_{q e}(\vec{Q}, t)$ is the intermediate scattering function for the incoherent quasielastic scattering and $I_{v i b}(\vec{Q}, t)$ is the vibration contribution. If the experiment is performed so that only neutrons scattered with energies close to the elastic scattering are collected, equation (37) combined with equations (29) and (33) gives, for one atom:

$$
I_{q e}(\vec{Q}, t)=e^{-Q^{2}\left\langle u_{0}^{2}\right\rangle} \cdot\left(I_{r t}^{i n c}(\vec{Q}, \infty)+I_{r t}^{t d}(\vec{Q}, t)\right)+e^{-Q^{2}\left\langle u_{0}^{2}\right\rangle} \cdot Q^{2}\left\langle u_{0}^{2}\right\rangle \cos \left(\omega_{0} t\right)[\ldots]
$$

This equation illustrates the fact that the Debye-Waller factor $\left(e^{-Q^{2}\left\langle u_{0}^{2}\right\rangle}\right)$ and the EISF $\left(I_{r t}^{i n c}(\vec{Q}, \infty)\right)$ are two fundamentally different quantities and that by measuring the incoherent elastic scattering, we measure their product.

\subsection{Concluding remarks}

The aim of this paper was first to show how spectroscopic means that bring a molecular system out of equilibrium could provide relevant information on the spontaneous fluctuations for the system at equilibrium. Thus classical statistical mechanics treatments, in particular Molecular Dynamics simulations, are of first importance for interpreting experimental data. Second, among other techniques, we have shown that neutron scattering spectroscopy is a nearly ideal technique to confront experiments and modelling. Indeed the only relevant dynamical variable is the position of the nucleus as a function of time. Therefore there are no unknown, or difficult to calculate, coupling functions. Neutron scattering 
experiment is the best technique to be used in synergy with computational methods, e.g. Monte Carlo (MC) and molecular dynamics (MD) simulations.

\section{References}

[1] G.L. Gouy, J. de Physique (2) 7:561 (1888)

[2] A. Einstein Ann.d.Phys. 17549 (1905)

[3] J. Perrin, Annales de Chimie et de Physique 19 (8ème série) (1909)

[4] P. Langevin, C. R. Acad. Sci. (Paris) 146530 (1908)

[5] N. Pottier in "Physique statistique hors d'équilibre : Processus irréversibles linéaires" Collection Savoirs Actuels EDP Sciences - CNRS Editions (2007)

[6] N. Pottier in "Nonequilibrium Statistical Physics : Linear Irreversible Processes" Oxford Graduate Texts, Oxford University Press (2009)

[7] D. A. McQuarrie in "Statistical Mechanics", University Science Books, Sausalito, California (2000)

[8] R. Kubo, “The fluctuation-dissipation theorem”, Rep. Prog. Phys. 29, 255-284 (1966).

[9] J.L. Barrat and R. Vuillemier, paper in this book

[10] F. Guillaume and M. Couzi, EDP Sciences, Collection SFN 10, 467 (2010)

[11] H. Schober, EDP Sciences, Collection SFN 10, 159 (2010)

[12] M. Bée, in "Quasielastic Neutron Scattering", Adam Hilger, Bristol and Philadelphia (1988)

[13] V. F. Sears, Neutron News, 3 (3) 29 (1992)

[14] P.A. Egelstaff in “Thermal Neutron Scattering”, Academic, New York (1971) 